\title{
Incoherent charge separation dynamics in organic photovoltaics
}

\author{
Shyamal K. K. Prasad ${ }^{\mathrm{a}}$, Joseph K. Gallaher, ${ }^{\mathrm{a}, \mathrm{b}}$, Alex J. Barker ${ }^{\mathrm{a}, \mathrm{c}}$, Han Young Woo ${ }^{\mathrm{d}}$, Mamatimin \\ Abbas $^{\mathrm{e}}$, Lionel Hirsch ${ }^{\mathrm{e}}$, Justin M. Hodgkiss ${ }^{\mathrm{a}}$ * \\ ${ }^{a}$ MacDiarmid Institute for Advanced Materials and Nanotechnology, and Victoria University of \\ Wellington, New Zealand; ${ }^{\mathrm{b}}$ School of Chemistry, University of New South Wales, Australia; ${ }^{\mathrm{c}}$ \\ Istituto Italiano di Tecnologia, Milan, Italy; ${ }^{\mathrm{d}}$ Department of Chemistry, Korea University, Seoul \\ 02841, Republic of Korea; ${ }^{\mathrm{e}}$ Univ. Bordeaux, IMS, UMR 5218, F-33400 Talence, France and CNRS, \\ IMS, UMR 5218, F-33400 Talence, France.
}

\begin{abstract}
There is mounting evidence that long-range charge separation determines the efficiency of organic photovoltaic cells, yet different mechanisms remain under debate. One class of proposed mechanism is ultrafast coherent long-range charge separation, and another is a slower process whereby charges incoherently hop apart with a transiently enhanced mobility due to morphology and disorder. Here, we use transient absorption spectroscopy to probe incoherent charge separation dynamics in two different ways. First, we use a family of polymers whose backbone structures allows us to compare 2phase donor-acceptor morphologies with 3-phase morphologies that feature an intermixed region. In the 3-phase system, we see pronounced spectral signatures associated with charges (holes) occupying the disordered intermixed region, and we track separation via biased charge diffusion to more ordered neat regions on the timescale of hundreds of picoseconds. Secondly, by resolving bimolecular charge recombination at high excitation density, we show that charge mobilities must be substantially enhanced on early timescales, which may be sufficient for separation to occur. Together, these measurements provide support for models of incoherent and relatively slow charge separation.
\end{abstract}

Keywords: Charge separation, organic photovoltaic, transient absorption, three phase, intermixed phase, mobility relaxation.

\section{INTRODUCTION}

In contrast to silicon cells, the functional components of organic photovoltaic (OPV) cells are $\pi$-conjugated molecular units that are responsible for the sequence of steps linking light absorption to photocurrent extraction. Owing to the more localized nature of states and less effective charge screening in organic semiconductors, the conversion of bound electron-hole pairs (either charge transfer states or excitons) to free charge carriers is crucial to the efficiency of OPV cells. Charge transfer from a donor polymer to an acceptor (e.g., fullerene) is necessary but insufficient to create photocurrent, as confirmed by the observation of geminate charge recombination (often radiative) via tightly bound interfacial charge transfer (CT) states. ${ }^{1}$ In spite of widespread acceptance that long-range charge separation is vitally important, the mechanism (or mechanisms) by which this happens remains under debate. Of the numerous mechanisms proposed from experimental and theoretical studies, we can identify two broad classes of mechanisms; coherent and incoherent charge separation processes.

Coherent charge separation invokes highly delocalized interfacial states in which the electron and hole components are sufficiently separated that they directly form free carriers upon relaxing into localized electron and hole states. One important feature of this mechanism is that long-range charge separation must occur on the ultrafast (femtosecond) timescale that such a coherent state exists. Bakulin et al examined the role of delocalized charge transfer states using a pump-push experiment, which revealed that relaxed CT states could contribute more photocurrent upon re-exciting to more delocalized CT states. ${ }^{2}$ Gelinas et al analyzed the dynamics of transient electroabsorption signals to quantify the velocity of charge separation in OPV blends and conclude that ultrafast charge separation over approximately $4 \mathrm{~nm}$ is

Physical Chemistry of Interfaces and Nanomaterials XV, edited by Artem A. Bakulin, Robert Lovrincic, Natalie Banerji, Proc. of SPIE Vol. 9923, 99231F · (C) 2016 SPIE · CCC code: 0277-786X/16/\$18 · doi: 10.1117/12.2238234 
enabled via coherent states in fullerene crystals. ${ }^{3}$ Kaake et al have further invoked the uncertainty principal in linking the ultrafast timecale of charge separation to intrinsic delocalization, ${ }^{4}$ albeit with theoretical reservations, ${ }^{5}$ and Chen et al showed that exciton states are rather delocalized on the ultrafast timescale that charge separation occurs in OPV blends. ${ }^{6}$ Theoretical modelling of OPV interfaces also confirms that delocalized hot CT states may be accessible, or even dominant, owing to the high density of delocalized CT states compared with localized states directly at the interface. ${ }^{7,8}$ However, a key study that is difficult to reconcile with the idea that hot CT states gate charge separation is Vanderwal et al's analysis of the photocurrent efficiency of relaxed CT states. ${ }^{1}$ Sensitive measurements correlating absorption strength and photocurrent efficiency in the optically weak sub-gap region showed that optically active CT states - even relaxed CT states - contributed photocurrent with approximately the same internal quantum efficiency as above-gap excitation. Since optically active states must have appreciable electron-hole overlap, other mechanisms beyond coherence are sought to explain their efficient charge separation.

Incoherent charge separation mechanisms, on the other hand, are characterized by slower separation of charges via multiple hopping steps between localized states. The question then remains what drives the process. Entropy favors charge separation, though not strongly enough to negate Coulomb binding alone. The groups of Kemerink and McGehee independently used simulations (supported by spectroscopy in some cases) so show that transiently enhanced charge mobility on early timescales would be sufficient to enable efficient incoherent charge separation via multiple hopping.9-11 Moreover, this mobility enhancement can be underpinned by energetic disorder, which is a defining characteristic of OPV materials. In this model, relaxation within an inhomogeneously broadened density of states results in high nonequilibrium charge mobilities. Another model of incoherent charge separation relates to the so-called 3-phase morphology that is observed for many efficient systems. Here, charges generated in intermixed phases are driven to separate to the respective pure donor and acceptor phases due to the greater stability of holes and electrons, respectively, in those phases. ${ }^{11-14}$
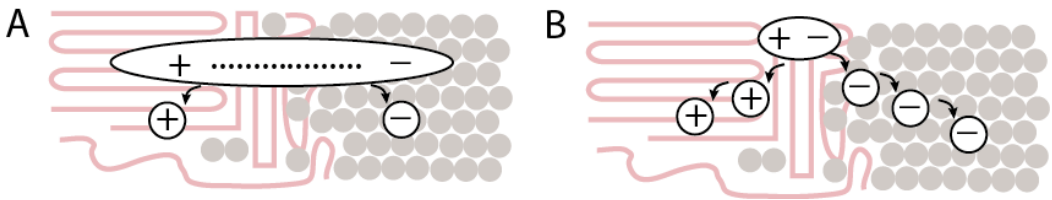

Figure 1. Schematic depiction of long-range charge separation via a) coherent delocalized states, and b) incoherent hopping processes.

Here, we apply transient absorption spectroscopy to examine incoherent charge separation dynamics in OPV blends. After employing a low temperature experiment to demonstrate that OPV efficiency is indeed linked to long-range charge separation, we explore charge separation in 3-phase OPV systems. Using signatures of holes occupying phase-pure versus intermixed regions, we reveal morphologically driven charge separation on the timescale of hundreds of picosconds. By measuring the excitation density dependence of non-geminate charge recombination dynamics, we also show that global kinetic models can only be satisfied when the bimolecular recombination constant - and by inference, the charge mobility - is strongly enhanced on early timescales. Our measurements provide experimental support for incoherent charge separation mechanisms in OPVs.

*Justin.Hodgkiss@vuw.ac.nz; phone+64 (0)4 4636983

\section{METHODOLOGY}

\subsection{Sample preparation}

Spectroscopic measurements were carried on using thin films of OPV active layers' spin coated onto fused silica substrates, but without contact electrodes. The film casting conditions were duplicated from those used in corresponding OPV devices, as per the cited references. Films were measured soon after deposition and housed inside a vacuum chamber during measurements to avoid the effects of air and moisture. 


\subsection{Transient absorption spectroscopy}

Transient absorption spectroscopy measurements were carried out using the system described in detail in references ${ }^{15}$ and ${ }^{16}$. The system is based on an amplified Ti:Sapphire laser running at $3 \mathrm{kHz}$, with supercontinuum probe shots read out on a shot-to-shot basis using visible and near-IR multichannel detectors. Key features and benefits of this setup for the current work include; i) the ability to capture TA spectra spanning from visible to near IR regions, thus providing clean signatures of excitons and charges in efficient OPV materials, ii) combination of mechanical and electronic delay configurations to achieve time resolution from $\sim 100$ fs to $\sim 300 \mu$ s with no gaps, spanning timescales of charge transfer, separation, and recombination, and iii) the ability to resolve differential transmission signals smaller than $10^{-5}$, enabling measurements from low excitation density as well as global analysis of non-geminate charge recombination over a wide range of densities.

\subsection{Data analysis}

Kinetic fitting for section 3.1 is described in detail in reference ${ }^{15}$. The kinetic decays in Section 3.3 employed a hybrid soft-hard modeling approach. Briefly, the monomolecular recombination regime was identified in a series of fluence dependent decay data by observing the fluence at which recombination becomes independent of fluence. Since these monomolecular dynamics were still non-exponential, there were fit using a cubic function ( $3^{\text {rd }}$ order polynomial), and this was used as the soft-modeled component against which bimolecular recombination competes. The fluence dependent series of charge recombination dynamics was then globally fit to a kinetic model that includes monomolecular (soft model) and bimolecular (density dependent) components, where the initial charge density for each measurement was fixed based on the known fluence, optical density, and film thickness. The global fitting procedure was carried out using a fixed bimolecular rate constant and also a bimolecular rate constant that is allowed to vary according to a power law decay to reflect mobility relaxation.

\section{RESULTS AND DISCUSSION}

\subsection{Long-range charge separation in organic photovoltaics}

We begin by presenting evidence, originally reported in reference ${ }^{15}$, that the yield of free charges in OPVs is determined by the probability of charge pairs initially achieving a separation on the order of 4 nanometers. By resolving charge recombination dynamics at low temperature $(10 \mathrm{~K})$, where activated charge diffusion is shut off and even well-separated charges must eventually recombine geminately, we are able to relate recombination dynamics to the distance distributions of charge pairs. Since charge recombination occurs in the tunneling regime, the recombination rate decays exponentially with electron-hole distance. Thus, distributions of tunneling recombination rates (on a logarithmic scale) are related to distance distributions via the tunneling decay constant, $\beta$.

Figure 2a shows the bimodal distribution of rate constants that fit the tunneling recombination dynamics of two P3HT:PCBM blends shown in Fig. 2b. A two-Gaussian rate model is used to fit the data in order to minimize the number of free parameters, however, it is important to note that even unconstrained multiexponential fits arrive at bimodal rate distributions with similar weightings that the Gaussian fits show in each component. The fitted rate distribution in Figure $2 \mathrm{a}$ is interpreted to mean that the rapidly recombining charges $\left(k_{\text {recomb }} \sim 10^{9} \mathrm{~s}^{-1}\right)$ correspond to closely separated charge pairs, or CT states, and the more slowly recombining phase (centered around $k_{\text {recomb }} \sim 10^{5} \mathrm{~s}^{-1}$ ) corresponds to wellseparated charges. The exponential decay of electron tunneling means that the logarithmic rate axis is directly proportional to electron-hole distance; applying a $\beta$ value of $\sim 0.3 \mathrm{~A}^{-17}$ results in the SC population being $\sim 3 \mathrm{~nm}$ greater separation than CT states (themselves likely $\sim 1 \mathrm{~nm}$ separation). Integrating each of these components gives the yields of $\mathrm{CT}$ and separated charges, denoted SC, as indicated on the plot. Figures $2 \mathrm{a}$ and $2 \mathrm{~b}$ reveal that annealing a P3HT:PCBM blend, which is known to improve efficiency, results in a greater fraction of SCs, suggesting that this analysis may be used to determine whether the long-range charge separation step underpins other blends.

In order to correlate long-range charge separation with free charge generation, we used the method developed by Howard et al to quantify the yield of free charges. ${ }^{17}$ By measuring room temperature charge recombination as a function of excitation density, geminate and non-geminate recombination is readily distinguished and the set of recombination kinetics can be globally fit to ascertain the fractions of charges that recombine via each channel. For our purposes, bimolecular recombination applies to free charges that would otherwise be swept out in a functioning device. Figures $2 \mathrm{c}$ and $2 \mathrm{~d}$ show the intensity dependent recombination kinetics obtained for the same P3HT:PCBM samples in Figures 2a and $2 \mathrm{~b}$, with the yields of free charges, $\Phi_{\text {free }}$, indicated on the plots. The free charge yields are remarkably similar to the 
SC yields measured at $10 \mathrm{~K}$, prompting us to extend the analysis to a wider range of materials. The fit employs fixed geminate and non-geminate rate constants, which likely accounts for slight deviations between the measured data and fits. We will return to this point in section 3.3.

Figure 2e shows the correlation between $\Phi_{\text {free }}$ and $\Phi_{\mathrm{SC}}$ across the range of materials measured, where each data point is underpinned by the series measurements exemplified in Figures 2a-d. The materials span blends with poor quantum efficiencies to higher performing blends. The striking correlation observed in Figure 2e shows that the yield of free (extractable) charges is determined the probability of charge pairs achieving sufficient initial separation. At low temperature, charge pairs are frozen at the sites where they thermalize and relax, which means that the relevant timescale implied by initial separation is that of charge relaxation. This measurement points to the importance of long-range charge separation, but does not distinguish between ultrafast coherent separation and incoherent hopping mechanisms, which both may occur at low temperature.
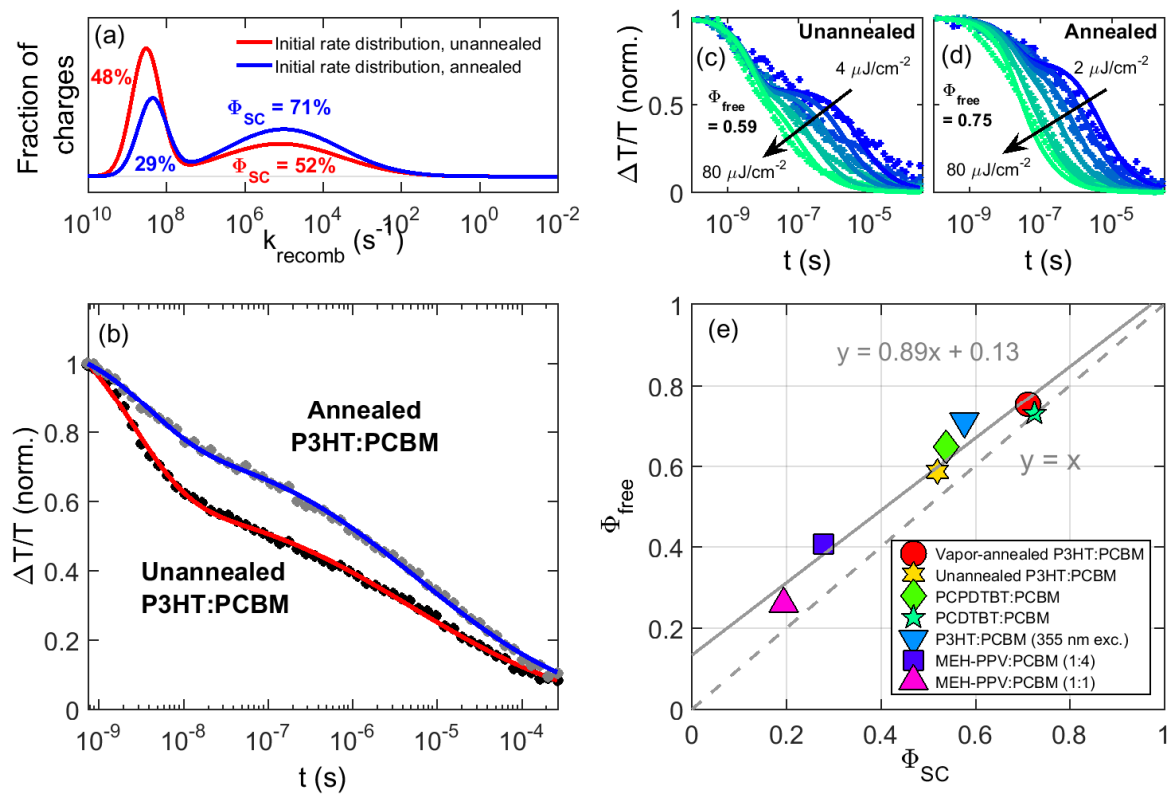

Figure 2. Adapted from reference [barker]. a) Distribution of recombination rate constants used to fit geminate charge recombination at $10 \mathrm{~K}$ for P3HT:PCBM samples shown in part (b). c) and d) Intensity dependent recombination at room temperature for the same P3HT:PCBM blends, fitted to the global kinetic model from reference [howard 2010]. e)

Correlation of $\Phi_{\mathrm{SC}}$ versus $\Phi_{\text {free }}$ for various blends according to the analysis in parts a)-d).

\subsection{Spectroscopically tracking charge separation in a three phase blend}

In order to test the hypothesis that a 3-phase system could drive charge separation, we compared transient absorption spectral dynamics in 2- and 3-phase systems comprised of two related low-bandgap polymers blended with PCBM. As reported in references ${ }^{18}$ and ${ }^{16}$, PTTBT forms a highly crystalline microstructure. Blends of PTTBT and PCBM form a 2-phase morphology whereby the polymer adopts the microcrystalline structure of neat films, without dissolving PCBM to form intermixed phases. PTBT, on the other hand, differs from PTTBT by a configuration of monomer units that induces backbone curvature and makes the polymer more compatible with PCBM. References ${ }^{18}$ and ${ }^{16}$ confirm that PTBT:PCBM blends exhibit a 3-phase morphology, where the amount of intermixed phase can be tuned by co-solvent processing.

Figure 3a shows the TA spectral evolution for the 2-phase PTTBT:PCBM blend. The spectra exhibit ground state bleaching signals in the visible, along with an exciton-based photoinduced absorption peak around $0.9 \mathrm{eV}$ and chargebased photoinduced absorption peaks around $1.1 \mathrm{eV}$. The entire TA surface is readily fit using only two spectra (respectively associated with excitons and charges) that do not shift over time. The TA dynamics in Figure 3a are easily accounted as exciton-to-charge conversion (prompt and diffusion limited) on the early picosecond timescale followed by charge recombination. Charge recombination occurs from late picosecond into nanosecond timescales, which is captured via the photoinduced absorption decay kinetics shown in Figure 3c. The rapid timescale of charge recombination approximately half within just 2 nanoseconds - reflects substantial geminate recombination of bound charge pairs that 
would not be extractable in a device, which is consistent with the comparatively poor efficiency (2.6\% PCE) ${ }^{18}$ of PTTBT:PCBM devices.

The TA spectral evolution for the 3-phase blend shown in Figure 3b reveals several important differences to the 2-phase blend that can account for the higher efficiency $(5.6 \% \mathrm{PCE})^{18}$ of the 3-phase system. First, the higher initial amplitude of the charge-based photoinduced absorption peak near $1.2 \mathrm{eV}$ reflects a higher fraction of prompt charge generation in strongly intermixed donor-acceptor phases. Second, the position of this absorption peak red-shifts over time to around $1.1 \mathrm{eV}$ at $500 \mathrm{ps}$. These near-IR absorption shifts are correlated with dynamic changes to the ground-state bleach peak shape, whereby the 0-0 vibronic peak becomes more pronounced over time. From this, we conclude that the spectral dynamics reflect migration of charges (hole polarons) from disordered to more ordered polymer regions in which polymer chains have greater extension, longer conjugation length, and more J-aggregate character. This conclusion is supported by observing how the shift magnitude is affected by solvent additives, and also by considering sensitization experiments that exclude the possibility that triplet exciton formation could cause spectral shifts. ${ }^{16}$ The polaron photoinduced absorption shift dynamics are plotted in Figure 3d. The 3-phase blend exhibits pronounced shifting dynamics throughout the picosecond timescale, whereas the 2-phase blend undergoes no appreciable shift. Moreover, the charge recombination kinetics for both blends shown in Figure 3c show that while substantial recombination happens within the first $2 \mathrm{~ns}$ for the 2-phase blend, recombination does not commence until after this timescale for the 3-phase PTBT blend. Considering the charge recombination dynamics (Figure 3c) in light of the spectral shift dynamics (Figure $3 \mathrm{~d}$ ), we conclude that charge migration from intermixed to phase pure regions (as depicted in the inset of Figure 3b) accounts for the suppressed charge recombination and higher photovoltaic efficiency of the 3-phase PTBT blend.
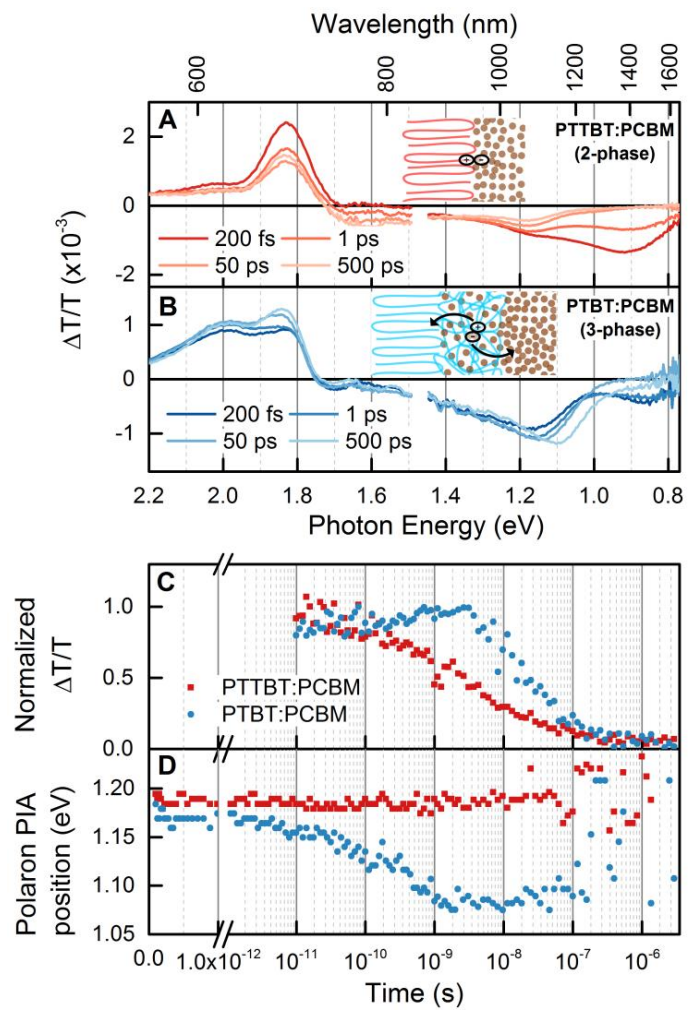

Figure 3. Transient absorption measurements of (A) PTTBT:PCBM, and (B) PTBT:PCBM thin films showing spectral slices at indicated time delays following $100 \mathrm{fs}$ excitation at $532 \mathrm{~nm}$. (C) Normalized recombination kinetics were tracked by integrated over the polaron PIA peak $\left(0.9-1.4 \mathrm{eV}\right.$ ) for both polymer:PCBM blends (excitation fluence $\sim 5 \mathrm{Jcm}^{-2}$ in both cases). Spectral overlap of polarons with excitons is avoided by excluding the dynamics before $10 \mathrm{ps}$, at which time excitons have decayed. (D) Analysis of the polaron PIA peak dynamics tracks the migration of holes from the intermixed domains into the phase pure domains in the 3-phase blend, which is absent in the 2-phase polymer:fullerene blend. 


\subsection{Mobility relaxation probed by bimolecular charge recombination dynamics}

In order to probe whether incoherent charge separation may be assisted by enhanced non-equilibrium charge mobilities on early timescales, we closely examined bimolecular charge recombination dynamics, which can be considered a proxy for charge mobility according to Langevin theory. By analyzing charge recombination dynamics over a wide range of excitation fluence, we were interested in whether recombination dynamics could be described using a single fixed recombination rate constant (i.e., a constant charge mobility), or whether there was evidence that the bimolecular rate constant decays with time (reflecting charge mobility relaxation). Our experiment included high fluences that were not supposed to mimic solar fluences but were sufficient to markedly accelerate recombination and thereby probe mobilities from early nanosecond timescales.

We applied this analysis to the PTTBT:PCBM and PTBT:PCBM blends discussed in section 3.2 above. The intensity dependent recombination data was limited to times beyond $\sim 2 \mathrm{~ns}$, noting that geminate recombination reduces the charge population by approximately a half prior to this for the PTTBT blend. Figures $4 \mathrm{a}$ and $4 \mathrm{~b}$ show that charge recombination is accelerated in both blends at high fluence - a signature of free charges. The recombination dynamics were fit using a hybrid hard-soft model described in the methodology section, in order to account for the non-exponential first order dynamics at low fluence. After soft-modelling the low fluence kinetics, the series' of fluence dependent dynamics were first fit using a single free parameter - the bimolecular rate constant, $k_{\mathrm{bi}}$, according to;

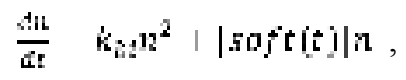

where $\mathrm{n}$ is the charge density, for which the initial value, $\mathrm{n}_{0}$, is fixed according to the known fluences, optical density, and film thickness. The fits according to equation 1 are shown as dashed lines in Figure 4. Deviation from the measured data is apparent for both data sets, particularly for the PTTBT:PCBM blend (Figure 4a). Specifically, while the fits appear adequate at early times, they decay too fast at longer timescales, suggesting that the real bimolecular rate constants are in fact reduced at longer times.

Assuming that the intrinsic recombination rate of encounter charge pairs remains constant, the apparent decay in the bimolecular rate constant must be linked to dynamic reduction in average charge mobility. Charge mobility in a low mobility system with Gaussian disorder is expected to relax according to a power-law. ${ }^{19,20}$ Thus, we adapted equation 1 with the addition of a power-law term, a, to describe the effect of mobility relaxation on $k_{\mathrm{b}}$, as follows;

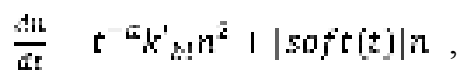

where the time-dependent bimolecular rate constant is recovered as;

$$
k_{b i}=t^{-a} k_{b i}^{\prime}
$$

Fits to both series of data using equation 3 are shown as the solid lines in Figures $4 \mathrm{a}$ and $4 \mathrm{~b}$. The fits reproduce the data very well over the entire time and fluence ranges. The fitting improvement can be quantified by comparing the $\mathrm{r}^{2}$-values for series with fixed and time-dependent $k_{\mathrm{bi}}$ values. For the PTTBT:PCBM blend, the $\mathrm{r}^{2}$-value increases from 91.6 to 97.7 upon allowing $k_{\mathrm{bi}}$ to relax with time. In the case of the PTBT:PCBM blend, the improvement is more subtle (96.5 to 97.1). Finally, time-dependent $k_{\mathrm{bi}}$ values were extract via equation 3 and plotted in Figure 4c. For the PTTBT blend, we see that $k_{\mathrm{bi}}$ decays by approximately two orders of magnitude from the early nanosecond to microsecond timescale, with a slightly weaker dispersion seen for the PTBT blend.

We conclude from this analysis that charge mobilites are indeed enhanced from their equilibrium values on early timescales, consistent with a similar bimolecular recombination experiment by Andersson $e t a l^{21}$ and other transient probes of charge mobility. ${ }^{22,23}$ It is premature to conclude that this mobility enhancement is sufficient to explain incoherent charge separation. In the future, we will need to extend this analysis to even earlier timescales on which charge separation is more likely to be occurring (as confirmed for these very blends in section 3.2). Sub-nanosecond measurements are likely to reconcile the apparent contradiction that the less efficient PTTBT blend exhibits more pronounced mobility relaxation than the more efficient PTBT blend; section 3 shows that the performances of these blends are distinguished via the charge separation/recombination competition on the sub-nanosecond timescale that is not captured in Figure 4. 

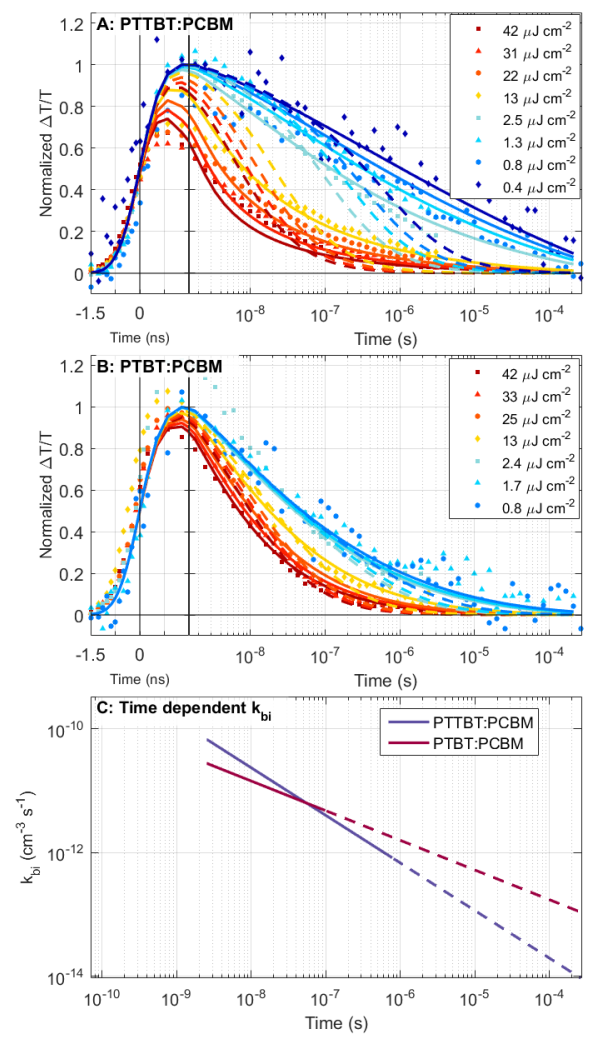

Figure 4. Series of fluence dependent recombination dynamics for A) PTTBT:PCBM and B) PTBT:PCBM. Fluences (with $532 \mathrm{~nm}, 600 \mathrm{ps}$ excitation) are indicated in the legend, and data is normalized by fluence. Dashed lines on both plots represent a global fit to equation 1, and solid lines represent fits to equation 2. C) Time-dependent $k_{\mathrm{bi}}$ values extracted (using equation 3) from the fits to equation 2, where the solid parts of the lines indicate the time regions where most of the resolved dynamics occur.

\section{CONCLUSIONS}

Using various configurations of fluence- and temperature dependent TA spectroscopy, we can draw several conclusions about charge separation in OPV films, and the plausibility of slow, incoherent processes. First, we show via tunneling recombination constants at low temperature that the initial charge separation step is a key determinant of free charge yields. Secondly, by comparing sub-nanosecond spectral dynamics in a 2-phase versus a 3-phase OPV blend, we examined the proposal that 3-phase blends may enhance charge separation via the cascaded energy landscape across the intermixed interfaces. We observe charges migrating from intermixed- to phase pure regions on sub-nanosecond timescales in the more efficient 3-phase blends, confirming the plausibility of this model. Finally, by analyzing fluence dependent recombination kinetics we find that data is not adequately fit using a fixed bimolecular recombination rate constant. Instead, the data is well described using a bimolecular rate constant that undergoes a time-dependent decay to reflect mobility relaxation. Assuming proportionality with the bimolecular rate constant, we find that mobility relaxes by two orders of magnitude from nanosecond to microsecond timescales, which may point to markedly higher mobilities assisting charge separation on earlier timescales. Collectively, our data supports proposals for incoherent, hopping-based, charge separation in OPVs. However, a broader question to keep in mind is whether one unifying charge separation mechanism describes all OPV cells, or whether several mechanisms can contribute with different weights to different material systems. 


\section{REFERENCES}

[1] Vandewal, K., Albrecht, S., Hoke, E. T., Graham, K. R., Widmer, J., Douglas, J. D., Schubert, M., Mateker, W. R., Bloking, J. T., et al., "Efficient charge generation by relaxed charge-transfer states at organic interfaces," Nat Mater 13(1), 63-68 (2013).

[2] Bakulin, A. A., Rao, A., Pavelyev, V. G., van Loosdrecht, P. H. M., Pshenichnikov, M. S., Niedzialek, D., Cornil, J., Beljonne, D., Friend, R. H., "The Role of Driving Energy and Delocalized States for Charge Separation in Organic Semiconductors," Science 335(6074), 1340-1344 (2012).

[3] Gelinas, S., Rao, A., Kumar, A., Smith, S. L., Chin, A. W., Clark, J., van der Poll, T. S., Bazan, G. C., Friend, R. H., "Ultrafast Long-Range Charge Separation in Organic Semiconductor Photovoltaic Diodes," Science 343(6170), 512-516 (2014).

[4] Kaake, L. G., Moses, D., Heeger, A. J., "Coherence and Uncertainty in Nanostructured Organic Photovoltaics,” J. Phys. Chem. Lett. 4(14), 2264-2268 (2013).

[5] Mukamel, S., "Comment on 'Coherence and Uncertainty in Nanostructured Organic Photovoltaics',' J. Phys. Chem. A 117(40), 10563-10564 (2013).

[6] Chen, K., Barker, A. J., Reish, M. E., Gordon, K. C., Hodgkiss, J. M., "Broadband Ultrafast Photoluminescence Spectroscopy Resolves Charge Photogeneration via Delocalized Hot Excitons in Polymer:Fullerene Photovoltaic Blends," J. Am. Chem. Soc. 135(49), 1850218512 (2013).

[7] Caruso, D., Troisi, A., "Long-range exciton dissociation in organic solar cells," Proceedings of the National Academy of Sciences 109(34), 13498-13502 (2012).

[8] Savoie, B. M., Rao, A., Bakulin, A. A., Gélinas, S., Movaghar, B., Friend, R. H., Marks, T. J., Ratner, M. A., "Unequal Partnership: Asymmetric Roles of Polymeric Donor and Fullerene Acceptor in Generating Free Charge," J. Am. Chem. Soc. 136(7), 2876-2884 (2014).

[9] van Eersel, H., Janssen, R. A. J., Kemerink, M., "Mechanism for Efficient Photoinduced Charge Separation at Disordered Organic Heterointerfaces," Adv. Funct. Mater. 22(13), 2700-2708 (2012).

[10] Howard, I. A., Etzold, F., Laquai, F., Kemerink, M., "Nonequilibrium Charge Dynamics in Organic Solar Cells,” Adv. Energy Mater. 4(9), 1301743 (2014).

[11] Burke, T. M., McGehee, M. D., "How High Local Charge Carrier Mobility and an Energy Cascade in a Three-Phase Bulk Heterojunction Enable $>90 \%$ Quantum Efficiency," Adv. Mater. 26(12), 1923-1928 (2013).

[12] Westacott, P., Tumbleston, J. R., Shoaee, S., Fearn, S., Bannock, J. H., Gilchrist, J. B., Heutz, S., deMello, J., Heeney, M., et al., "On the role of intermixed phases in organic photovoltaic blends," Energy Environ. Sci. 6(9), 2756 (2013).

[13] Collins, B. A., Li, Z., Tumbleston, J. R., Gann, E., McNeill, C. R., Ade, H., “Absolute Measurement of Domain Composition and Nanoscale Size Distribution Explains Performance in PTB7:PC 71BM Solar Cells," Adv. Energy Mater. 3(1), 65-74 (2012).

[14] Groves, C., "Suppression of geminate charge recombination in organic photovoltaic devices with a cascaded energy heterojunction,” Energy Environ. Sci. 6(5), 1546 (2013).

[15] Barker, A. J., Chen, K., Hodgkiss, J. M., "Distance Distributions of Photogenerated Charge Pairs in Organic Photovoltaic Cells,” J. Am. Chem. Soc. 136(34), 12018-12026 (2014).

[16] Gallaher, J. K., Prasad, S. K. K., Uddin, M. A., Kim, T., Kim, J. Y., Woo, H. Y., Hodgkiss, J. M., "Spectroscopically tracking charge separation in polymer : fullerene blends with a three-phase morphology," Energy Environ. Sci. 8(9), 2713-2724 (2015). 
[17] Howard, I. A., Mauer, R., Meister, M., Laquai, F., "Effect of Morphology on Ultrafast Free Carrier Generation in Polythiophene:Fullerene Organic Solar Cells,” J. Am. Chem. Soc. 132(42), 14866-14876 (2010).

[18] Lee, W., Kim, G.-H., Ko, S.-J., Yum, S., Hwang, S., Cho, S., Shin, Y.-H., Kim, J. Y., Woo, H. Y., "Semicrystalline D-A Copolymers with Different Chain Curvature for Applications in Polymer Optoelectronic Devices," Macromolecules 47(5), 1604-1612 (2014).

[19] Bässler, H., "Charge Transport in Disordered Organic Photoconductors a Monte Carlo Simulation Study," physica status solidi (b) 175(1), 15-56, WILEY VCH Verlag (1993).

[20] Mozer, A. J., Dennler, G., Sariciftci, N. S., Westerling, M., Pivrikas, A., Österbacka, R., Juška, G., "Time-dependent mobility and recombination of the photoinduced charge carriers in conjugated polymer/fullerene bulk heterojunction solar cells," Phys. Rev. B 72(3), 035217-10 (2005).

[21] Andersson, L. M., Melianas, A., Infahasaeng, Y., Tang, Z., Yartsev, A., Inganäs, O., Sundström, V., "Unified Study of Recombination in Polymer:Fullerene Solar Cells Using Transient Absorption and Charge-Extraction Measurements," J. Phys. Chem. Lett. 4(12), 2069-2072 (2013).

[22] Melianas, A., Pranculis, V., Devižis, A., Gulbinas, V., Inganäs, O., Kemerink, M., "Dispersion-Dominated Photocurrent in Polymer:Fullerene Solar Cells," Adv. Funct. Mater. 24(28), 4507-4514 (2014).

[23] Ponseca, C. S., Jr., Yartsev, A., Wang, E., Andersson, M. R., Vithanage, D., Sundström, V., "Ultrafast Terahertz Photoconductivity of Bulk Heterojunction Materials Reveals High Carrier Mobility up to Nanosecond Time Scale," J. Am. Chem. Soc. 134(29), 11836-11839 (2012). 